\title{
Thermodynamic properties of water in the conditions of the planet Mars.
}

Timur Khokhoev Elbrusovich

Address: 362040 Russia, Vladikavkaz, Butyrina st. 10/54

Tel: + 7-918-836-50-90

Mail: timurhohoev@gmail.com

In the reports of the space agencies NASA and ESA on missions carried out on the planets of the solar system, in particular on Mars, the discovery of water is reported. The discovery of water on Mars is of fundamental scientific importance. Also, this issue becomes more and more urgent in the light of the planned manned flights to Mars, because the water balance in the human body is $65 \%$. The purpose of this article is a detailed study of water and its properties in the conditions of Mars.

This article discusses the possibility of changed thermodynamic properties of water. For this, such phenomena are being studied as: the presence of water in the Mars mesosphere and the covered clouds with a rover Curiosity. Also, the article nominates the interpretation of some phenomena and incidents, such as: depressurization of the cuvette comparison of the Marshode Curiosity, the failure of the Mars-3 apparatus, the presence of liquid water on Plutonon, the relief on the Crater Hale and in the Polar region of Mars, the cause of the origin of the winds on Mars, the work of the Bura Insight and the presence of water on the satellite of Jupiter in a liquid form.

\section{Water in the mesosphere.}

In 2019, researchers from the Moscow Institute of Physics and Technology (MIPT) and the Institute for Research of the Solar System of the Society named Max Planck modeled the scheme of movement of water in the atmosphere of Mars [1]. According to the model, we can talk about the presence of water vapor at an altitude of $60 \mathrm{~km}$, where the temperature is much lower than the average temperature of the surface of Mars, i.e. below $-75^{\circ}$ C. Referring to the phase diagram of state (Fig. 2), we can say that with the standard thermodynamic properties of water, it cannot be at the indicated altitudes in the atmosphere of Mars in liquid or gaseous form. It follows from this that the published data of the Institute for Research of the Solar System of the Society named Max Planck and MIPT require additional study of the properties of liquid water of the planet Mars.

\section{Clouds on Mars.}

On May 17, 2019, NASA's Curiosity rover captured drifting clouds (Fig. 1) $31 \mathrm{~km}$ above the surface of Mars [2]. In search of a method for the formation of these clouds, 2 most likely assumptions were put forward:

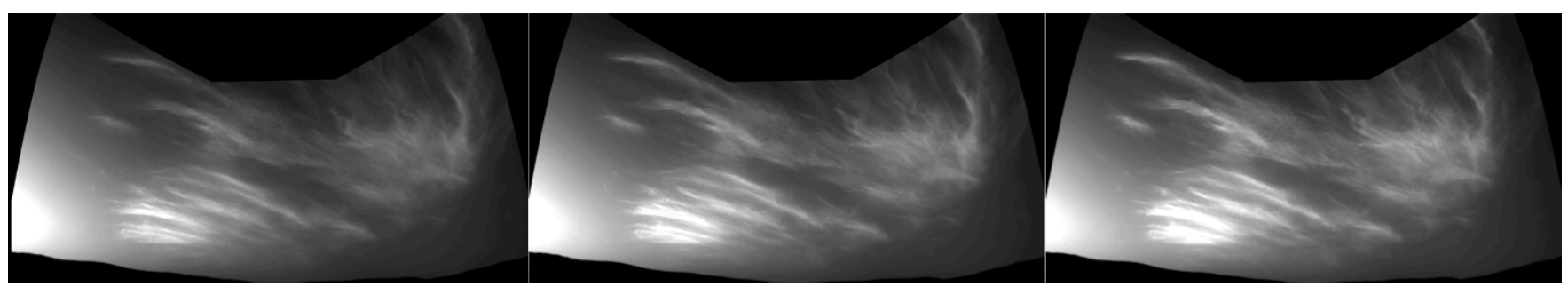

(Fig. 1)

1) Clouds are composed of particles of carbon dioxide condensed at low temperatures at this altitude.

2) Clouds are made of water.

When considering the model of these clouds as a consequence of the condensation of carbon dioxide, it is worth considering that the atmosphere of Mars is $95 \%$ carbon dioxide. The image shows that these clouds are condensed locally and formed from matter that makes up only a small part of the atmosphere. If conditions were right for $\mathrm{CO}_{2}$ condensation, the clouds would appear denser and occupy more of the sky than what is shown in the image, since almost all of the atmosphere in this area would have condensed. From which it can be assumed that these clouds are not made of $\mathrm{CO}_{2}$. 
Considering the model of these clouds, the composition of which is water with its classical properties, it is necessary to turn to the phase diagram of the state of water (Fig. 2). On Mars, the average temperature is $-70^{\circ} \mathrm{C}$ and the pressure is $530 \mathrm{~Pa}$. The diagram shows that with these parameters, the water should be in a solid state of aggregation. Even taking into account that at a given altitude of $30 \mathrm{~km}$, the pressure is much lower, about $20 \mathrm{~Pa}$ (Fig. 3), at this temperature water is a solid body and cannot reach the ice-gas phase boundary. Those. Under these conditions, it is impossible to have an equilibrium system with a transition from a gaseous state to a solid and / or vice versa, therefore, in this case, precipitation will be the expected phenomenon. In fact, no precipitation was observed.
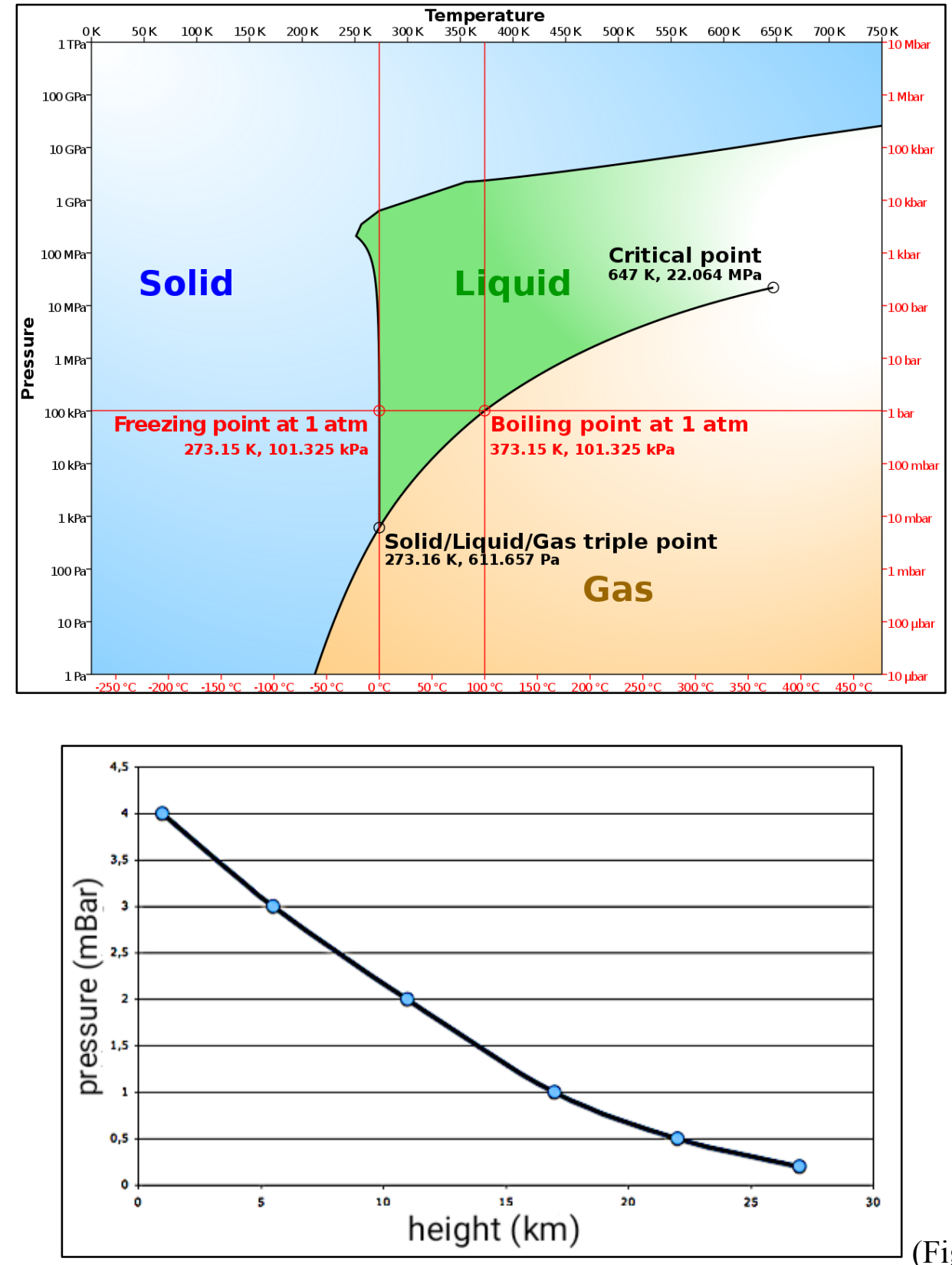

The purpose of the article is to analyze the collected scientific data and identify patterns taking into account the behavior of water as a chemical in comparison with known substances with similar molecular structures and their properties.

The above contradictions can be explained as follows. If we compare the thermodynamic properties of water with substances similar in molecular structure, namely, with hydrogen compounds and non-metals of group VI of the periodic table of elements, we can see that under terrestrial conditions water abruptly falls out of the regular series of physical properties of substances similar in structure (Fig. 4). 
1) H2Te: $\mathrm{t}_{\text {melt }}=-2{ }^{\circ} \mathrm{C}$; $\mathrm{t}_{\text {boil }}=-48^{\circ} \mathrm{C}$

2) H2Se: $t_{\text {melt }}=-40{ }^{\circ} \mathrm{C} ; \mathrm{t}_{\text {boil }}=-65^{\circ} \mathrm{C}$

3) H2S: $\mathrm{t}_{\text {melt }}=-60^{\circ} \mathrm{C}$; $\mathrm{t}_{\text {boil }}=-88^{\circ} \mathrm{C}$

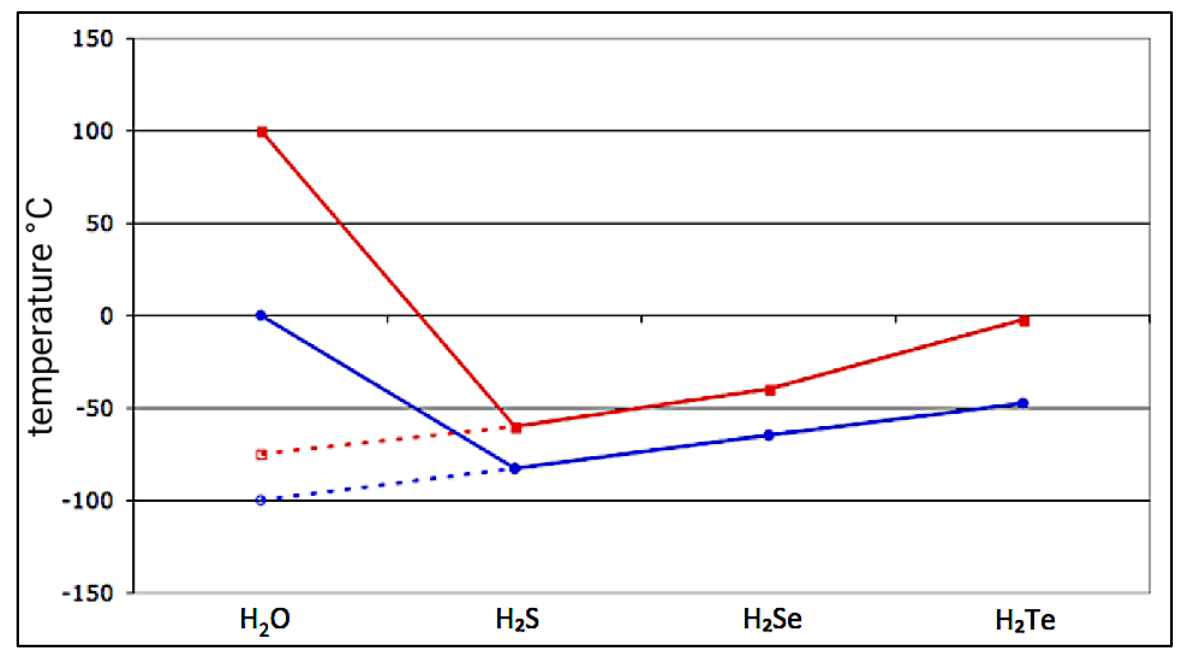

(Fig. 4)

If the general laws acted on water, then the boiling point would be $-75^{\circ} \mathrm{C}$ and the melting point $-100^{\circ}$ C. This discrepancy is explained by the presence in water of intermolecular hydrogen bonds between hydrogen and a strong oxidizing agent (oxygen); in the case of subsequent elements of group VI of the main subgroup, hydrogen bonds are not observed due to the insufficiently strong oxidizing properties of chalcogenes. Taking into account the similar dynamics of the phase transitions of chalcogenhydrogens with respect to the pressure and temperature parameters, it is possible to simulate the phase diagram of the state of water taking into account the general laws. Keeping the geometry of the curves denoting the boundaries of the phases and making a correction for the boiling and melting points at atmospheric pressure, a phase diagram of the state of water was modeled in the absence of hydrogen bonds (Fig. 5).

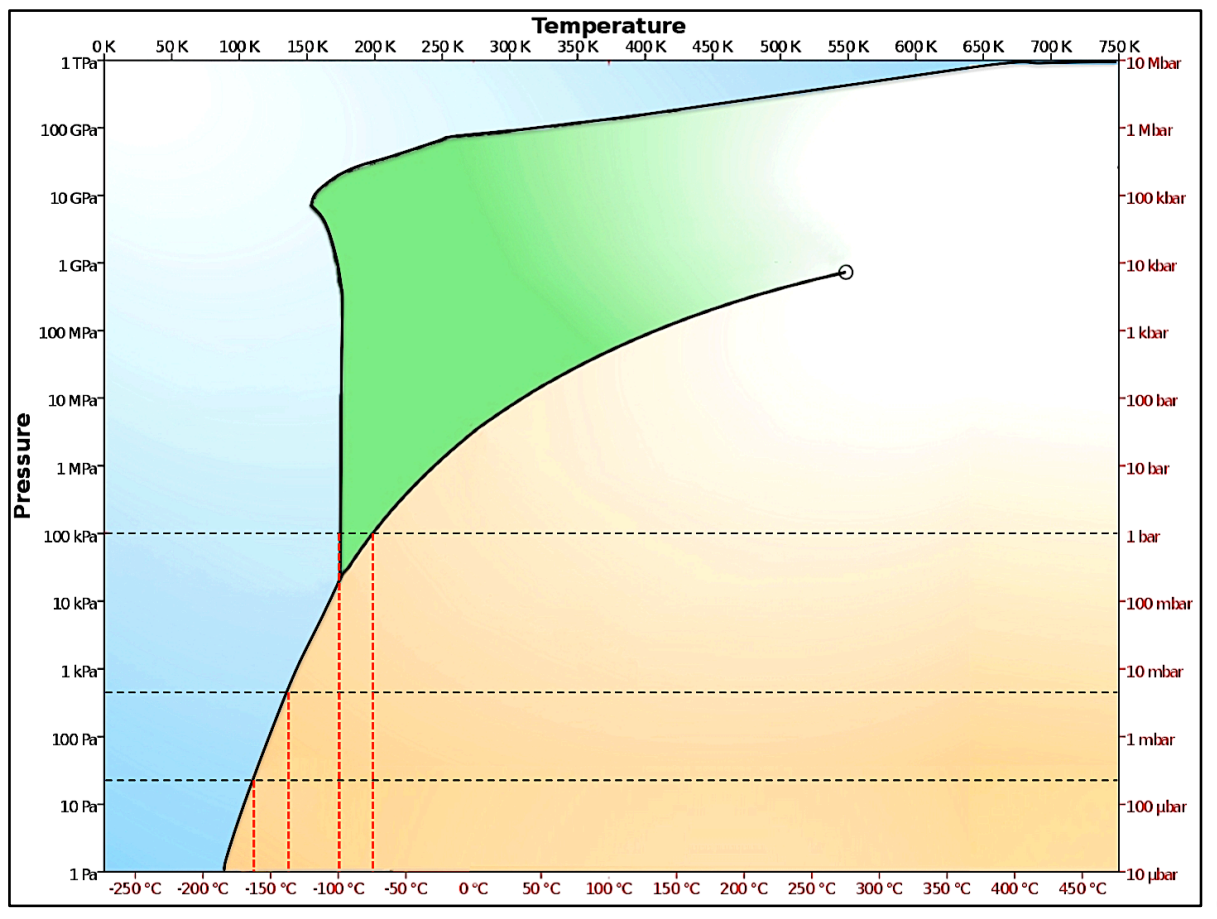

If we talk about water on Mars as a substance without hydrogen bonds, the study of some phenomena from this point of view gets their explanation.

The presence of water in a gaseous state in the mesosphere where the temperature is below $-75^{\circ} \mathrm{C}$ is quite acceptable when considering water with altered thermodynamic properties. The diagram shows 
that at this pressure, water is in a gaseous state starting from a temperature of $-170^{\circ} \mathrm{C}$, which confirms and supplements the model of water movement, modeled in the study of the MIPT and the Institute for Research of the Solar System of the Society named Max Planck.

The assumption of the absence of hydrogen bonds details the theory of the water composition of the clouds captured by the Curiosity rover. The clouds are at a height where the average pressure is $20 \mathrm{~Pa}$. According to the diagram, we can say that water at this pressure will be in a gaseous state at temperatures above $-165^{\circ} \mathrm{C}$. Taking into account the decrease in temperature with increasing altitude, it can be assumed that the temperature in that area was close to $-165^{\circ} \mathrm{C}$ and the water was in gas-ice phase equilibrium. The solid state is confirmed by the icy glare in the clouds, and the abrupt sublimation is confirmed by the absence of precipitation.

When interpreting some phenomena on Mars, taking into account the assumptions put forward above, one can explain the nature of their formation and course.

\section{Cuvettes of the Curiosity rover.}

The Curiosity rover landed on Mars in 2012. The purpose of the mission was to conduct a comprehensive analysis of the Martian soil. According to the article [5], during one of the soil analyzes, the rover's laboratory was able to detect organic matter in the soil of Mars. Later, from an interview with Dani Glavin (Deputy Director of Strategic Sciences, Solar System Research Department), it was suggested that the presence of organics on Mars was the result of depressurization of control samples of organics which included water that occurred as a result of landing, . NASA experts have put forward the assumption that the depressurization occurred due to the shaking of the rover during landing. In parallel, the depressurization of capsules with organic samples from the Curiosity rover may have the following explanation. When the rover left the boundary of the conditions affecting the formation of hydrogen bonds, the boiling point was $-75^{\circ} \mathrm{C}$. However, due to low temperatures in outer space, water did not go into a gaseous form. After the capsules entered the atmosphere of Mars, the temperature increased and the transition to the gaseous phase, in a limited volume, the pressure increased 900 times, which caused the destruction of the control cells intended for spectral analysis and the ingress of terrestrial organic matter onto the surface of Mars.

\section{Mars - 3.}

In the context of the analysis of this issue, we can consider the device AIS Mars - 3 launched on May 28, 1971 in the USSR. This article [6] says that 1.5 minutes after the descent vehicle landed, data transmission began, which lasted for 20 seconds. After that, the signal was lost. There are no explanations as to what caused the failure of the device, but given the absence of hydrogen bonds on Mars, the explanation of what caused the failure of the Mars - 3 apparatus may be the assumption that water was part of the hydraulic mixture, which was one of the components in the design of the apparatus. When entering the atmosphere of Mars, the same process occurred as with the capsules of the Curiosity rover. The result of this process was an increase in the pressure in the hydraulic system up to 900 atmospheres, which, with a high degree of probability, could lead to an explosive destruction of the hydraulics and the apparatus itself.

\section{Water on Pluto.}

The unusual behavior of water relative to the generally accepted point of view is manifested not only on Mars. The study [7] states that on January 20, 2016, the presence of liquid water under the surface of Pluto was detected. It is known that the temperature of Pluto's surface is $-210^{\circ} \mathrm{C}$, which does not allow the presence of water in liquid form. An attempt to explain this phenomenon was made in the article. It reports that Pluto's planetary heat is conserved by the surface layer of methane hydrate, which acts as thermal insulation for Pluto's energy. However, if we give a similar example on our planet, then the presence of methane gas hydrates in the Black Sea rushes into account. Observations show that hydrates concentrate on the seafloor rather than float to the surface. When considering the "isolation" of Pluto by analogy with the Earth, one can come to the conclusion that methane hydrates on Pluto cannot form a surface layer. At a surface temperature of $-210^{\circ} \mathrm{C}$, the presence of water under the surface with the 
absence of hydrogen bonds and, as a consequence, with a melting and boiling point of $-100{ }^{\circ} \mathrm{C}$ and $-75^{\circ}$ $\mathrm{C}$ respectively (at atm. Pressure), is more likely than the presence of water with Earth thermodynamic properties, with the melting point and boiling point of $0^{\circ} \mathrm{C}$ and $100^{\circ} \mathrm{C}$ and with the presence of hydrogen bonds, which should remain in liquid form due to the supposed heat-insulating layer of some compound, for example, from an instrumentally undetected layer of methane hydrate.

\section{Relief at Hale Crater.}

According to the article [8], in 2015, in a message from the Mars Reconnaissance Orbiter, it was said about the discovery of relief on the slope of the Hale crater, which was classified as traces of water flows descending from the rock formations, by analogy with the terrestrial ones (Fig. 6). Later, in March 2017, NASA experts stated a different assumption in an article [9]. It was said that the diverging trails were formed by gases emanating from the interior of the planet. The first theory of water descending from the top of the crater was rejected because the amount of ice reserves in that area did not allow theformation of stable water flows.

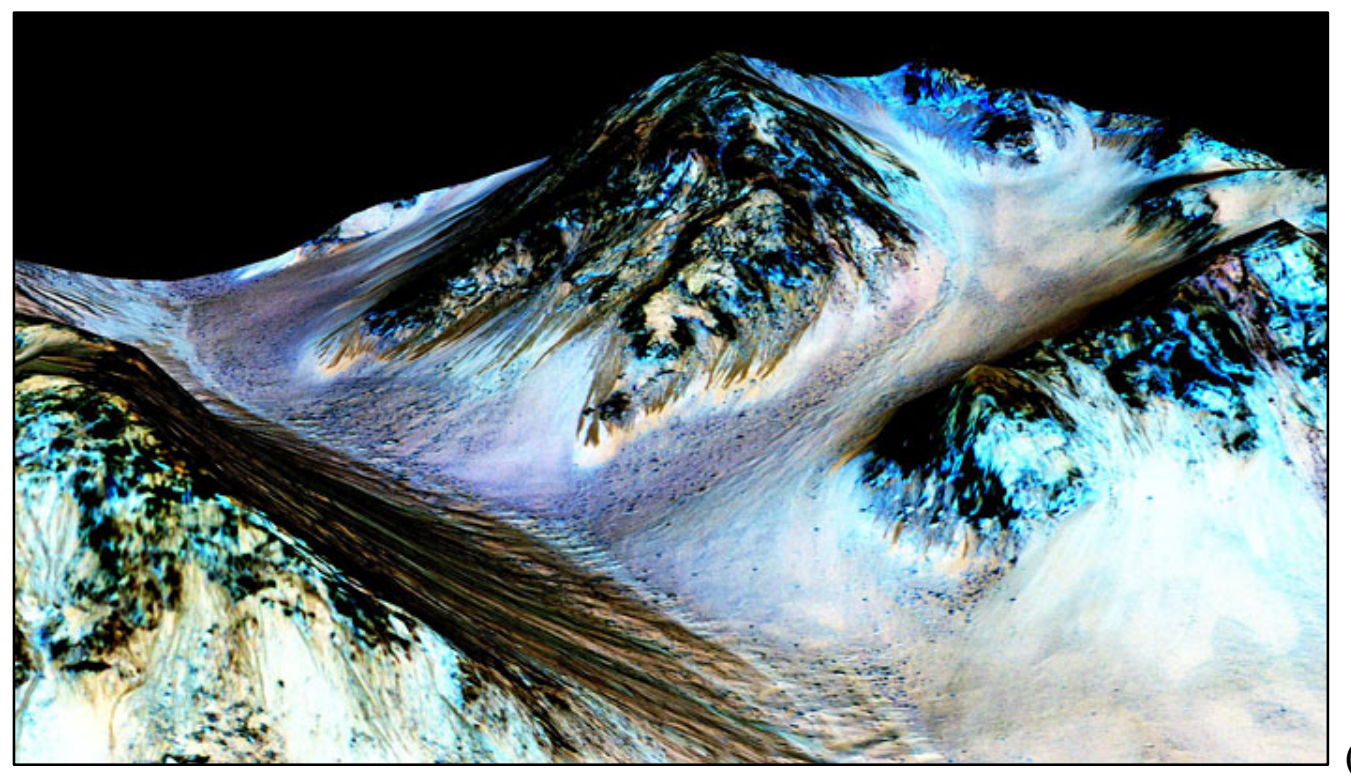

This conclusion leads to the question: "What is the source of gas escaping from under the ground in such a volume that allows you to leave a trail of sliding dunes?" Let's try to analyze the geological processes taking place in Hale Crater. The presence of a fine sandy mass was confirmed, forming talus along the slopes of the crater. Under the surface layer, it has been proven that ice is found in a volume insufficient for the formation of water flows capable of providing the appearance of the surface sand layer. Meanwhile, the traces of descending sand dunes absolutely prove that their demolition was provided by a sufficient volume of the working fluid. And therefore, a working fluid was considered to be a certain gas emanating from the core of Mars. Observations of the study of the relief of that area do not show the presence of volcanic activity, which could be the source of this gas. However, looking at this phenomenon from the point of view proposed in this article, it can be assumed that the thermodynamic properties of subsurface ice may allow it to be the source of this working fluid (gas) in the summer season, which will push the dunes down the crater. Since, according to the proposed theory, the absence of hydrogen bonds will allow ice to increase the volume of the working fluid by a factor of 900 under negative temperatures during the transition from a solid to a gaseous state, this amount meets the necessary requirements for the formation of characteristic traces of flowing sand dunes.

\section{Relief in the polar region of Mars.}

According to the description of the photograph (Fig. 7) taken by the Trace Ctas Orbited station [10], at the north pole of Mars there are areas with uncharacteristic relief for Mars, classified as traces from the release of carbon dioxide from under the surface through sublimation and, as a result, excess pressure under the layer carbon dioxide. Carbon dioxide is undoubtedly a substance with a sublimation temperature $\left(-78^{\circ} \mathrm{C}\right)$ suitable for the local conditions $\left(-70^{\circ} \mathrm{C}\right)$. 


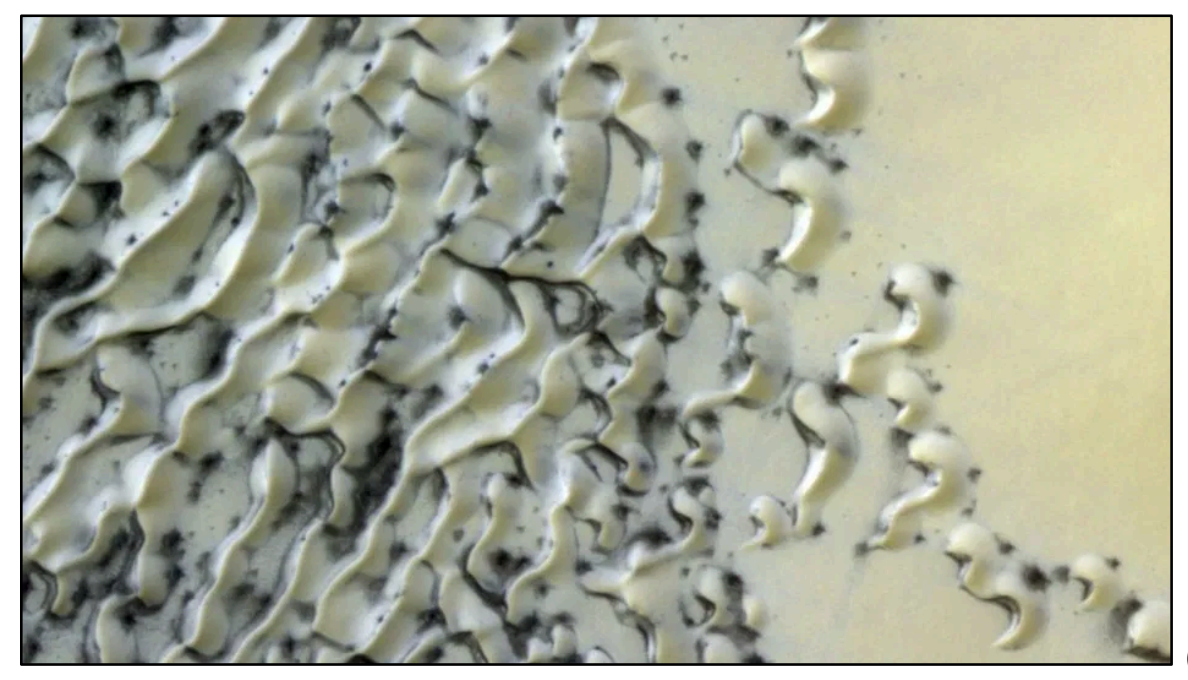

(Fig. 7)

But if we take into account the above properties of water, then it is likely that water is also the source of these relief formations, and given the reduced pressure and, as a result, the sublimation temperature of $140^{\circ} \mathrm{C}$ (Fig. 5), water can form these relief forms in more cold season.

\section{Winds on Mars.}

On Mars, strong winds are observed in the polar regions. They arise in places with a large accumulation of ice, at a temperature suitable for sublimation. The probable process can proceed as follows. During the phase transition of ice into gas or gas into ice, the volume of the working fluid changes by a factor of 900 and, as a consequence, the pressure changes, which can be the cause of strong winds.

On the surface of Mars, winds in the form of a tornado of small sizes are often observed (Fig. 8) [11]. In terrestrial conditions, tornadoes with a pronounced center arise during atmospheric activity and a developed cloud system. Due to the low warming up of the Mars atmosphere by the Sun, such phenomena are not observed on the planet. On Earth, tornadoes form from a cloud, developing downward and most often in a single number. On the contrary, Martian vortices develop from the surface upwards. This suggests that the source that gives energy to the vortices is on the surface, and not in the atmosphere of the planet. Visually, the wind data is analogous to the phenomenon of the pyroclastic effect on Earth. This phenomenon occurs during volcanic eruptions. When lava comes into contact with water, there is a sharp evaporation and the formation of winds in the form of a tornado. Moreover, similar winds are observed during forest fires and when burning oil products. These cases are generalized by the fact that the source of tornadoes is on the surface. And by analogy, this is another confirmation that the source of energy for the Martian vortices is in the soil of Mars. Eddies are observed in multiple numbers where theoretically there are ice accumulations and surface heating is favorable for phase transitions of Martian ice. It is worth noting that the equatorial zone receives much more heat, but these phenomena are absent there, since there are no ice deposits.

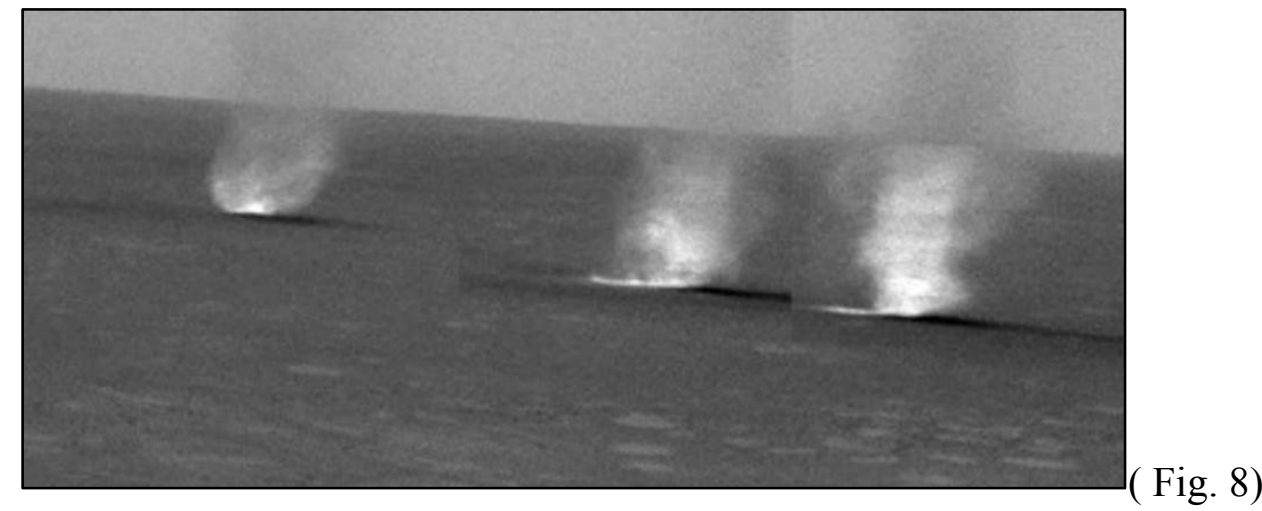

What could be the reason for the absence of hydrogen bonds. 
Mars and Pluto have a very weak magnetosphere compared to Earth. It can be assumed that when that core of Mars was hot, its mobility and rotation provided a stable magnetosphere and, as a result, the presence of liquid water, as evidenced today by numerous dry river beds. The reason for the sinking of Mars may be the termination of the flow of radiogenic heat from the decay of isotopes in the core of Mars (Fig. 9). On Mars, by analogy with the earth, the heat flux is determined not by the instantaneous decay of nucleids in the core, but by the decay that took place some time ago, a time sufficient for the radiogenic heat to reach the surface.

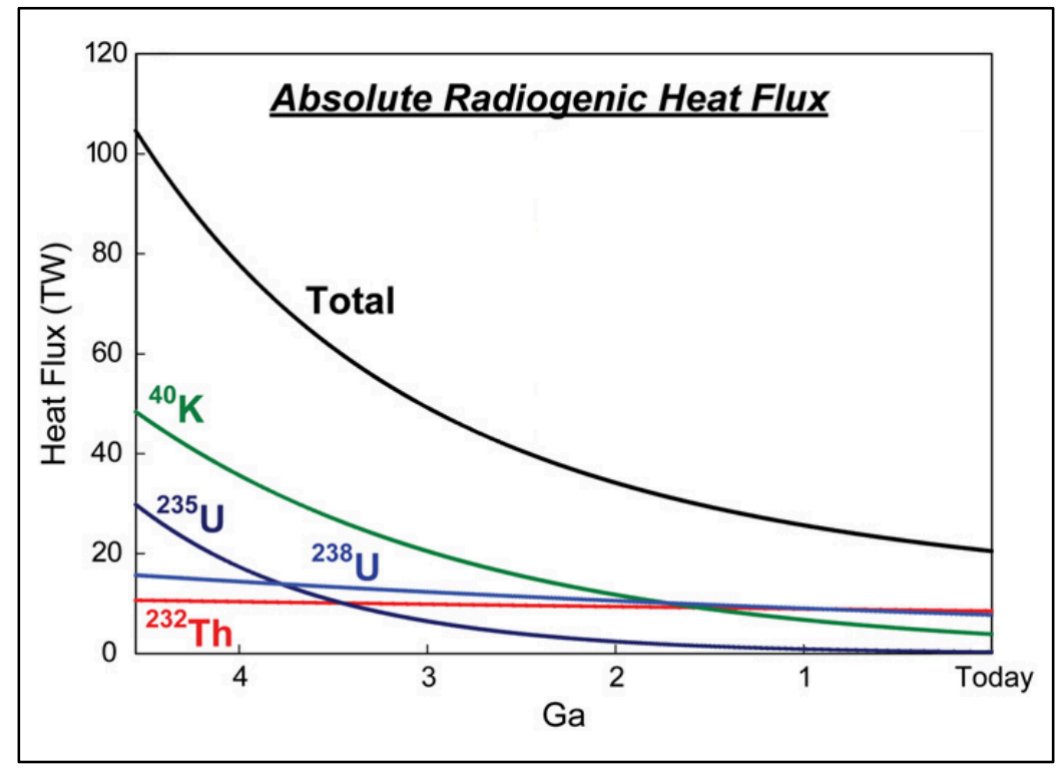

(Fig. 9 [12])

The isotopic composition of Mars and Earth, like those of planets formed simultaneously from the same mass, is the same. But the difference lies in the fact that on Earth today the peak of radiogenic decay heat only reached the surface, and on Mars, due to the smaller radius and faster heat flux reaching the surface, the heat from the peak of the decay of isotopes in the mantle reached the surface much earlier, which ensured the cooling of the planet and dissipation energy into space. And the heat that previously provided Mars with a liquid core, a stable magnetosphere and, as a consequence, the possibility of hydrogen bonding near water, has now dissipated in space, this has led to a change in the thermodynamic properties of water, which forced the water masses of Mars to concentrate on the polar regions, drying out the equatorial zone planet.

The following phenomena can be considered as confirmation of the influence of the magnetic field on the formation of hydrogen bonds.

\section{Probe Insight.}

On November 26, 2018, the InSight station successfully landed on the surface of Mars on the Elysium Planitia. One of the mission's goals was to study the soil of Mars with the help of a drill, which was planned to be deepened under the surface of Mars by impulse impacts to a depth of more than 5 meters. On February 28, 2019, the drill of the station began to work, but came across a solid obstacle. On March 2 , the drill was resumed for four hours, but it did not progress further, so it was decided to suspend drilling for two weeks [13]. Moreover, it was reported that during the impulse impacts, the probe slowly rose from the soil. The final attempt to deepen the boer was carried out with the help of a manipulator, which pressed the drill, after which the drill went a little deeper. As a result, the drill went deep by about $50 \mathrm{~cm}$. The explanation of why the final result did not meet the planned expectations can be as follows. The drill was driven by an electromagnet located in its metal case, which means that the magnetic moment was transmitted along the entire length of the drill. When the unit was activated, the gases of water without hydrogen bonds that were in the soil under the influence of a magnetic field began to form hydrogen bonds, and water with the usual thermodynamic properties appeared under the drill. If in this area the temperature is sufficient for water without hydrogen bonds to be in a gaseous state (Fig. 5), then "classical" water will be in the form of ice (Fig. 2). Some time after the start of drilling, enough ice 
accumulated for the drill to resist. Moreover, when the drill was inactive for a long time during which the ice could pass into its previous gaseous form, the drill was deepened with the help of a manipulator that did not create such a strong magnetic field under the base of the drill.

\section{Water on Europe.}

As an example confirming the influence of the magnetic field on hydrogen bonds, the satellite of Jupiter - Europe, which overlaps the magnetic field of Jupiter, has a hot core and, according to astronomical observations, has a large amount of water in liquid form [14].

\section{Conclusion.}

The article discusses the assumption of the absence of hydrogen bonds in water and changing its thermodynamic properties in the Mars planet. From this point of view, it turned out to interpret the nature of some phenomena on Mars that had no definite explanation before that.

This question undoubtedly needs further learning and analysis. It is necessary to fully understand the behavior of water on under Mars. This issue acquires even greater relevance in the light of the planned piloted flights to Mars, despite the fact that the water balance in the human body is $65 \%$. 


\section{Literature:}

1. https://agupubs.onlinelibrary.wiley.com/doi/10.1029/2019GL082839

2. https://mars.nasa.gov/resources/22497/curiositys-three-frame-mosaic-of-clouds/

3. https://ru.wikipedia.org/wiki/\%D0\%A4\%D0\%B0\%D0\%B9\%D0\%BB:Water_phase_diagram.gif

4. https://journals.ametsoc.org/view/journals/atsc/50/21/1520-

0469_1993_050_3625_mvatas_2_0_co_2.xml

5. https://www.science.org/doi/10.1126/science.360.6393.1054

6. https://en.wikipedia.org/wiki/Mars_3

7. https://arxiv.org/pdf/1606.04840v2.pdf

8. https://www.nature.com/articles/ngeo 2412

9. https://www.nature.com/articles/s41598-017-07453-9

10. https://www.esa.int/ESA_Multimedia/Images/2019/09/North_polar_dunes_on_Mars

11. https://www.nasa.gov/image-feature/jpl/pia21482/curiosity-observes-whirlwinds-carryingmartian-dust

12. https://www.sciencedirect.com/science/article/abs/pii/S0012821X08007711

13. https://mars.nasa.gov/news/8612/mars-insight-lander-to-push-on-top-of-the-mole/?site=insight

14. https://www.nasa.gov/feature/goddard/2019/nasa-scientists-confirm-water-vapor-on-europa 